\title{
Radio Resource Management Techniques for High Throughput Satellite Communication Systems
}

\author{
Svilen Dimitrov*, Stefan Erl*, Benjamin Barth*, Stephan Jaeckel ${ }^{\dagger}$, Argyrios Kyrgiazos ${ }^{\ddagger}$ and Barry G. Evans ${ }^{\ddagger}$ \\ ${ }^{*}$ German Aerospace Center (DLR), 82234 Wessling, Germany, e-mail: \{svilen.dimitrov, stefan.erl, benjamin.barth\}@dlr.de \\ ${ }^{\dagger}$ Fraunhofer Heinrich Hertz Institute, 10587 Berlin, Germany, e-mail: stephan.jaeckel@hhi.fraunhofer.de \\ ${ }^{\ddagger}$ University of Surrey, Centre for Communication Systems Research, GU27XH, UK, e-mail: \{a.kyrgiazos, b.evans\}@surrey.ac.uk
}

\begin{abstract}
In this paper, interference-aware radio resource management (RRM) algorithms are presented for the forward and return links of geostationary orbit (GEO) high throughput satellite (HTS) communication system. For the feeder link, satellite-switched smart gateway diversity is combined with two scheduling methods to improve the feeder link availability in rain conditions. For the user link, interference-aware scheduling (IAS) for the forward link and scheduling based on multipartite graph matching for the return link are shown to enable full frequency reuse (FR) multi-beam satellite systems. The performance assessment of scheduling algorithms is carried out in a system-level simulator with realistic channel models and system assumptions. The improvements of the system capacity and user rates are evaluated.
\end{abstract}

Index Terms-Satellite communications, radio resource management, full frequency reuse, co-channel interference mitigation.

\section{INTRODUCTION}

The traffic demand for satellite broadband is expected to grow 6-fold by 2020 [1]. Next generation high-throughput satellite (HTS) systems need to offer both higher throughput and higher data rates, while decreasing the cost per transmitted bit. Terabit/s satellite communication technologies are studied in the European BATS project [1]. In order to meet the capacity demands, a multi-beam HTS system needs to employ a very high reuse over the coverage area, combined with intelligent resource management mechanisms to alleviate the resulting co-channel interference (CCI) [2].

Appropriate scheduling of the physical layer (PL) frames on the time-frequency resources, i.e. carriers and time slots, in the beams of a satellite system can help mitigate the CCI. This approach is suitable for burst mode time division multiplexing (TDM) in the forward link or time division multiple access (TDMA) in the return link. However, the variable-length DVBS2 [3] frame structure with continuous mode transmission is not suitable for time-frequency scheduling. Fortunately, the recently introduced extension to the standard, i.e. DVB$\mathrm{S} 2 \mathrm{X}$ [4], includes the superframe concept, where the superframes can pack different modulation and coding schemes

This paper has been accepted for publication in the Proceedings of the European Conference on Networks and Communications 2015 (EUCNC 2015), 29 June - 02 July 2015, Paris, France, DOI: 10.1109/EuCNC.2015.7194063.

(C) 2015 IEEE. Personal use of this material is permitted. Permission from IEEE must be obtained for all other uses, in any current or future media, including reprinting/republishing this material for advertising or promotional purposes, creating new collective works, for resale or redistribution to servers or lists, or reuse of any copyrighted component of this work in other works.
(MODCODs), and they have a fixed duration. If alignment of the superframes on the carriers is assumed, the forward link can also accommodate burst mode transmission, as is the case with the return link DVB-RCS2 [5] standard. While the return link inherently benefits from scheduling to mitigate the CCI, the forward link can only benefit, if a number of the interfering beams are switched off at a given time step or if there are available free carriers and time slots, i.e. the system is not under full load. Novel CCI-aware scheduling algorithms have been introduced in [2]. In this paper, the performance of the most promising solutions is evaluated for full FR in the forward and return satellite links of a practical HTS system.

The rest of the paper is organized as follows. Section II presents the scheduling algorithms and their performance in the forward and return links. Section III concludes the paper.

\section{RESOURCE MANAGEMENT ALGORITHMS FOR THE FORWARD AND RETURN LINKS}

Very high capacity multi-beam HTS systems require a high number of beams over the coverage area and very high reuse of the available system resources, i.e. frequency bands and time slots. This, however, reduces the distance between the co-channel beams and leads to an increased CCI. Interferenceaware RRM is considered as the key to the realization of very high resource reuse HTS systems.

In the forward link, an intended user experiences CCI from the co-channel beams that transmit data to their users on the same carrier and time slot. Therefore, if the system is under full load, i.e. all the beams are active on all the resources, the CCI experienced by an intended user is constant and cannot be alleviated through scheduling. However, if there are free resources in the system, vulnerable users with low carrierto-noise ratio $(\mathrm{C} / \mathrm{N})$ can be scheduled on resources which are lightly used across the beams. Alternatively, a number of interfering beams can be switched off at a given time step. These approaches help increasing the signal-to-noiseand-interference ratio $(\mathrm{C} /(\mathrm{N}+\mathrm{I}))$ and the achievable data rates.

In the return link, based on the association of users in the co-channel beams to share a system resource, different CCI is experienced by the users. Through optimization of the user associations and scheduling over the available resources, the CCI can be minimized and the user throughput maximized. As opposed to the forward link, appropriate scheduling improves the CCI, even if the system is under full load. 
In the following, the studied RRM techniques are discussed in detail, and their performance is evaluated in the BATS system scenario [1].

\section{A. Satellite-switched smart gateway diversity for feeder link}

Considering a time switched payload, more flexibility in terms of interconnections between feeder and user links is introduced into the system. The time that each feeder link is connected with a user link may vary over time. The update instructions are transmitted from a network control centre (NCC) to the satellite, and the onboard scheduler is thus updated. A framework which takes into account the atmospheric conditions of each gateway plus the traffic demands of the beams and decides the number of time slots that each gateway is connected to a user beam is proposed allowing a match to the instantaneous user demands with the offered capacity of the gateways. The dwelling time of a gateway to a user beam can be tuned such as to minimize the capacity losses, keeping as low as possible the number of gateways in the system whilst providing the required availability. Two objective functions have been considered herein: 1) one that maximises the product of the satisfaction ratios (offered traffic/traffic demands) for the sake of load balance; and 2) another that maximises the minimum satisfaction ratio for the sake of fairness.

In a frame period, a beam is served by $N$ active gateways multiplexed over time. Such a configuration can be regarded as $N$-ary site diversity. It is obvious that as the number of gateways that serve a beam increases, the probability that this beam experiences an outage decreases significantly. A multibeam satellite system with $N$ feeder links and $M$ user links is considered. Let's define as $C_{i j}$ the instantaneous average (over spot beam's area) offered capacity (bps) from gateway $i$ to user beam $j$, where $i=1,2, \ldots, N$ and $j=1,, 2, \ldots, M$. Let the requested traffic per frame period (bits) for beam $j$ be defined as $R_{j}$. The offered traffic (bits) to a beam $j$ is defined as $B_{j}$, and it can be expressed as:

$$
B_{j}=\sum_{i=1}^{N}\left(C_{i j} X_{i j}\right) T_{s},
$$

where $X \in \mathbb{Z}^{N \times M}$. The $X_{i j}$ represents the number of time slots that feeder link $i$ is connected with user link $j$, and $T_{s}$ denotes the duration of a time slot. Moreover, the offered data rate $C_{i j}$ is subject to the channel conditions at each gateway:

$$
C_{i j}=B_{c} \eta_{i j}
$$

where $\eta_{i j} \in \mathbb{R}$ represents the achievable spectral efficiency, and $B_{\mathrm{c}}$ is the carrier bandwidth. The traffic losses are defined as $L=\sum_{j=1}^{M} \max \left\{R_{j}-B_{j}, 0\right\}$. The objective is to match the feeder link transmission rates with the user beam requests. Herein, two different objective functions are studied. The first one method is considered for load balancing:

$$
\underset{X}{\operatorname{maximize}} \prod_{j=1}^{M} \frac{B_{j}(X)}{R_{j}},
$$

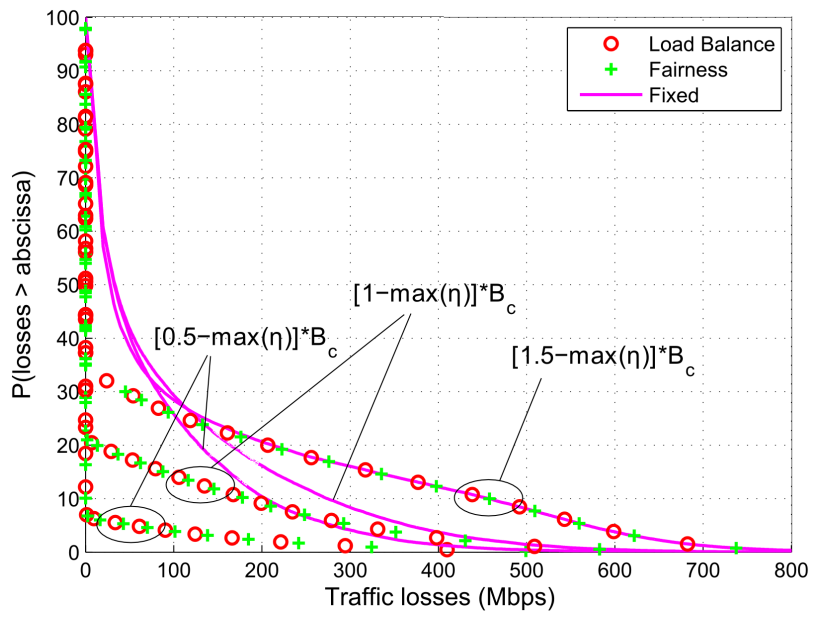

Fig. 1: CCDF of traffic losses for a fixed scheduling and the proposed algorithms for 4 gateways in a cluster.

\begin{tabular}{|r|rrrr|}
\hline$\Delta[\%]$ & \multicolumn{4}{|c|}{ Unsatisfied traffic [Mbits] } \\
\hline \# GES & 100 & 200 & 300 & 400 \\
\hline 2 & 0 & 0 & 0 & 0 \\
3 & 16.8 & 10.5 & 8.5 & 6.8 \\
4 & 26.2 & 18.5 & 14.6 & 10.8 \\
5 & 34.6 & 24.3 & 18.1 & 12.8 \\
6 & 38.33 & 26.6 & 19.25 & 13.23 \\
\hline
\end{tabular}

TABLE I: Reduction of traffic losses with respect to the case with 2 gateways in the cluster for a given unsatisfied traffic.

while the second method can be employed to improve fairness:

$$
\underset{X}{\operatorname{maximize}} \min _{1 \leq j \leq M}\left(\frac{B_{j}(X)}{R_{j}}\right) \text {. }
$$

Detailed description of these methods can be found in [6]. In this study, we present their effectiveness in the BATS system. A feeder link frame duration of $1 \mathrm{sec}$ is considered, comprising of 200 slots. Therefore, the requested and offered traffic can be defined in bps. Generally, for a broadband system, duration of a frame of $1 \mathrm{sec}$ can imply non-acceptable latency, if no traffic is offered to some beams. However, in the considered smart gateway approach, there is always a guaranteed connection for a user link to a feeder link during the frame period.

Fig. 1 shows the complementary cumulative distribution function (CCDF) of the traffic losses (bps) for three traffic scenarios, where the users traffic demands are drawn from a uniform distribution $[\{0.5,1,1.5\}-\max (\eta)]^{*} B_{c}$ (bps) for the fixed scheduling and the two proposed methods, considering 4 gateways in a cooperation cluster. Note that these distributions contain only the non-zero values, as a zero value represents a satisfied traffic request. The maximum achievable spectral efficiency, $\max (\eta)$, depends on the link budget, detailed for the BATS system in [2] for $B_{\mathrm{c}}=447.3 \mathrm{MHz}$. An important observation is the reduction of traffic losses due to the better match of feeder transmissions rates with the user beam demands. The probability of small losses decreases significantly compared to the fixed case, while the probability of large losses is also reduced. The effectiveness of the proposed methods is 


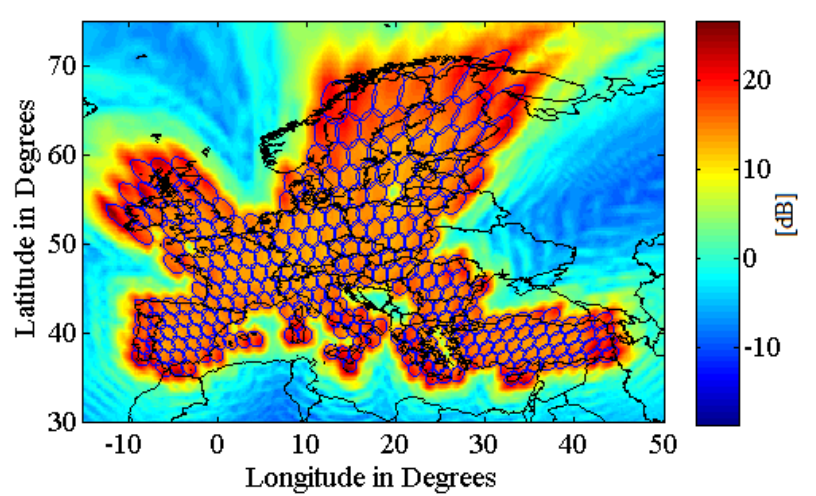

Fig. 2: C/I and beam layout of the BATS system with FR-4.

improved as the number of gateways in the cluster or the traffic variance increase. In TABLE I, the percentage reduction of the traffic losses, $\Delta$, with respect to the case with 2 gateways in a cluster is presented for a given value of unsatisfied traffic in the case of traffic requests within $[1-\max (\eta)]^{*} B_{c}$.

\section{$B$. Interference-aware scheduling for forward user link}

The IAS algorithm is designed for full FR. It utilizes partial channel state information (CSI) to reduce the number of interfering beams on the served users. The IAS disables neighbouring beams, if a served user cannot handle the interference caused by these beams. As a result, it is best suitable for burst mode beam hopping applications. The performance of the IAS is evaluated using the 302 beam layout specified in the BATS system [1], and it is compared to a frequency reuse 4 Round Robin (FR4-RR) scheme.

The structure of the IAS is composed of three parts. First, users send partial CSI, i.e. the received power from every beam. This is used in the second step to determine the serving beam and the beams which have to stay inactive, if the user receives data. Such beams are those that are received by the user with up to $6 \mathrm{~dB}$ less power than the serving beam. The $6 \mathrm{~dB}$ threshold was found to be optimal for the BATS system and might be different in other systems. The final step is the user scheduling described in the following.

1) Select Beam for Scheduling: This is based on two factors. First, the algorithm prefers beams close to already inactive beams. Second, beams which have been scheduled less often have priority. Hence, beams are selected more uniformly which improves the fairness.

2) Determine Permitted Users: Users which already have been scheduled are blocked until all other users in the same beam have been served. Furthermore, all users which would require that already scheduled beams are disabled are also blocked.

3) Select User for Transmission: For all remaining users, the achievable rate is calculated, assuming that not yet processed beams are inactive. For already scheduled users in other beams, the interference is increased. Hence, the rates for already scheduled users are updated, as well. Then, the user which increases the system capacity the most is selected. If there is no user in the

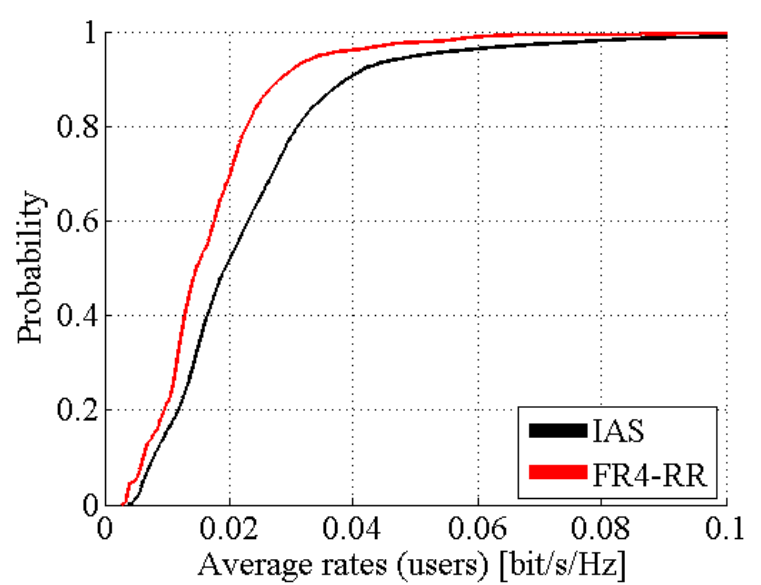

Fig. 3: Average rate per user in the BATS scenario.

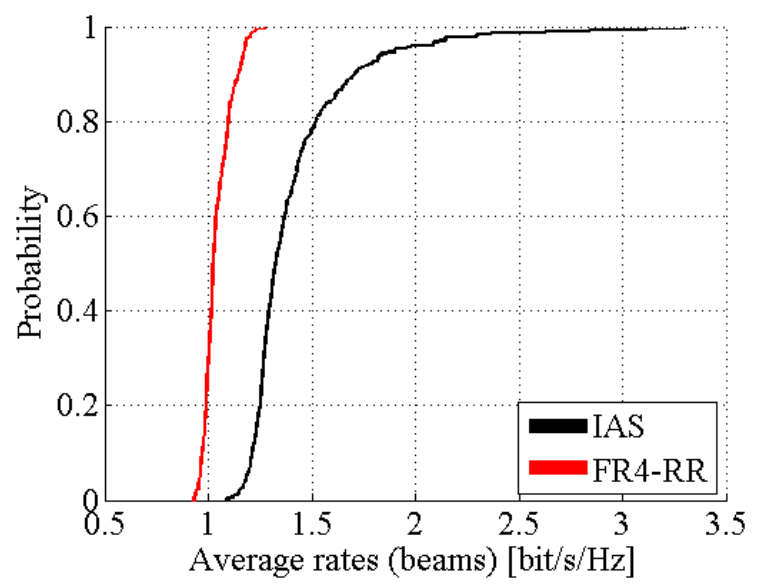

Fig. 4: Average rate per beam in the BATS scenario.

current beam that is able to increase the system rate, the beam is blocked and no user is scheduled.

4) Update the Processed Beams: The beams which have to stay inactive for the selected user are marked as blocked to ensure that the user can achieve a rate. The scheduling is done, when all beams are either scheduled or blocked.

In comparison, the FR4-RR scheduler serves all users in a beam consecutively and restarts, if all users have been served. In Fig. 2 the beam layout and the carrier-to-interference ratio $(\mathrm{C} / \mathrm{I})$ with FR-4 is shown. The C/I changes with the IAS and full FR depending on the user position and the activated beams. In the simulations, the $\mathrm{C} /(\mathrm{I}+\mathrm{N})$ ranges from $1.1 \mathrm{~dB}$ to $16 \mathrm{~dB} .17986$ users are placed on a $0.2^{\circ} \times 0.2^{\circ}$ grid inside the shapes of the participating countries. Realistic beam patterns are used to calculate the received power of the users. A noise power of $-82.4 \mathrm{dBm}$ and a transmit power of $47.4 \mathrm{dBm}$ per beam are assumed. The receive power of the users' strongest beam varies between $-74 \mathrm{dBm}$ and $-64.7 \mathrm{dBm}$ due to path loss and antenna gains of transmit and receive antenna. The channel is assumed to be perfectly known at the receiving user terminals (UTs), ideal synchronization and clear sky conditions are assumed. Users send their receiving power of each beam to a centralized hub which conducts the scheduling. The user rates $R$ are calculated by the Shannon function and 


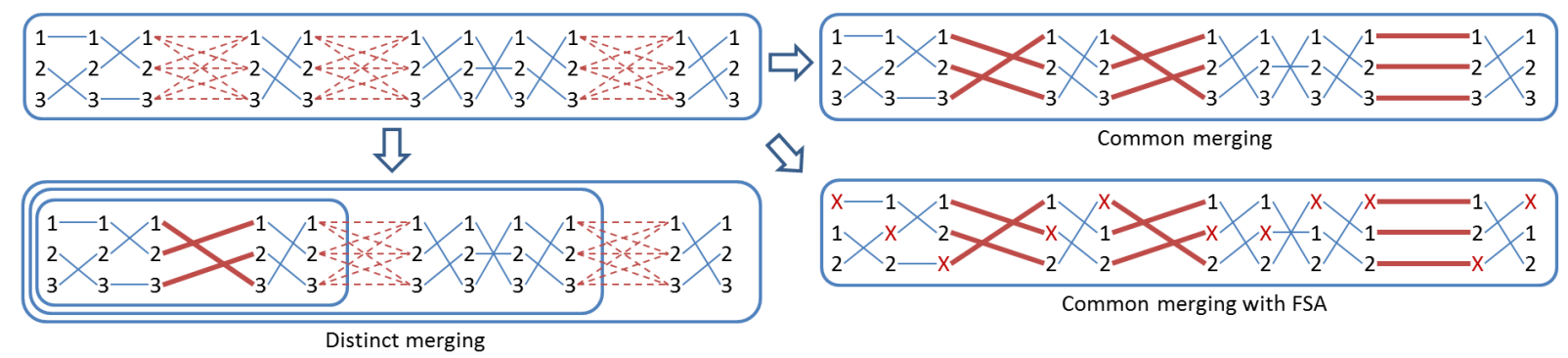

Fig. 5: Graph matching algorithms.

the $\mathrm{C} /(\mathrm{I}+\mathrm{N})$ :

$$
R=\mathrm{lb}\left(1+10^{0.1 \cdot \mathrm{C} /(\mathrm{I}+\mathrm{N})}\right) .
$$

The user rates are averaged over 10000 time slots. The data rates are normalized to the full system bandwidth of $2.9 \mathrm{GHz}$ in the Ka band forward user link. The cumulative distribution function (CDF) of the average rates of the users is shown in Fig. 3. The IAS is able to increase the minimum, maximum and mean data rate of the all users. Since TDM is employed, disabling of beams does not relate to an outage, but rather a delay in serving the traffic request. However, no noteworthy latency is observed compared to the FR4-RR. Both the IAS and the FR4-RR presented no $\mathrm{C} /(\mathrm{I}+\mathrm{N})$ values below the outage threshold of $\mathrm{C} /(\mathrm{I}+\mathrm{N})=-1.85 \mathrm{~dB}$ defined in the BATS system, therefore meeting the availability requirement of $99.7 \%$. In Fig. 4, the CDF of the average rate of the beams is presented. The scheduler offers significant increase of the average rates of the beams, where the beams with the highest gains are beams at the border of the coverage which offer better C/I conditions. The system rate is calculated by averaging the rates of the beams. The IAS is able to achieve an average system rate of $1.415 \mathrm{bit} / \mathrm{s} / \mathrm{Hz}$, while the FR4-RR only achieves an average system rate of $1.043 \mathrm{bit} / \mathrm{s} / \mathrm{Hz}$, which represents a gain of $35.7 \%$.

\section{Scheduling based on multi-partite graph matching for return user link}

Scheduling algorithms for full FR based on multi-partite graph matching have been presented in [7]. The goal is the maximization of the minimum user rates by allocating the system resources to the users in an optimal way, so that the CCI is minimized. To reduce the computational complexity, users are split into clusters. The assumption is made that users in second tier beams contribute only negligibly to the CCI. Therefore, a virtual 4-color scheme is applied, and the users in a color are scheduled randomly. These random cluster schedules are merged together by applying a minimum deletion algorithm and different graph matching techniques to find the optimum user association. The distinct merging is a greedy approach that iteratively merges two clusters at once. While this approach has low computational complexity, it implies the possibility of finding local optimum. This is overcome by the common approach, which finds the global optimum by merging all clusters in one step. A variation of both techniques is the Free Slot Assignment (FSA) method. Here, one slot per

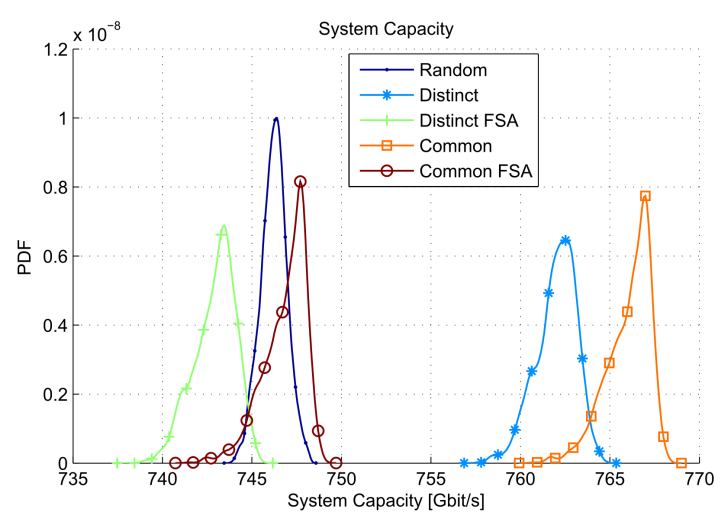

Fig. 6: System capacity with SIC.

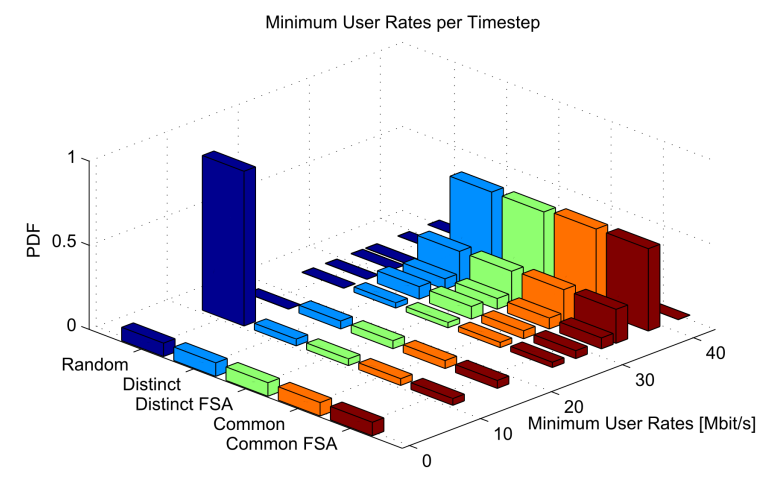

Fig. 7: Minimum user rates with SIC.

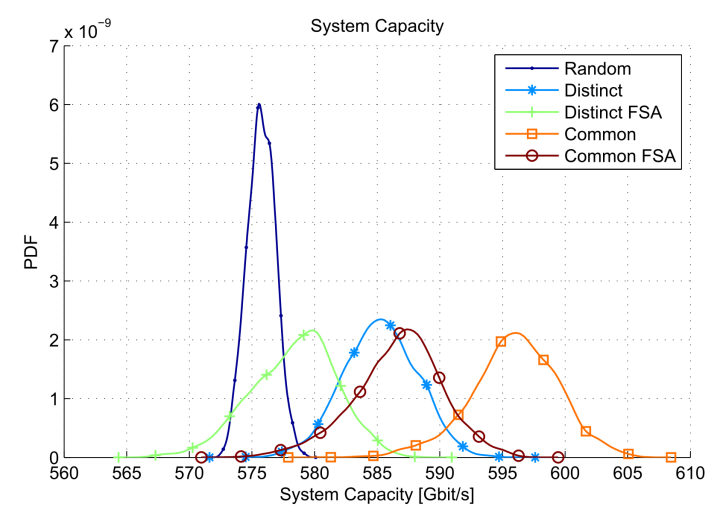

Fig. 8: System capacity without SIC.

schedule is left unallocated, which increases the flexibility in the scheduler to further optimize the schedule and minimize the CCI. The merging techniques are summarized in Fig. 5. 


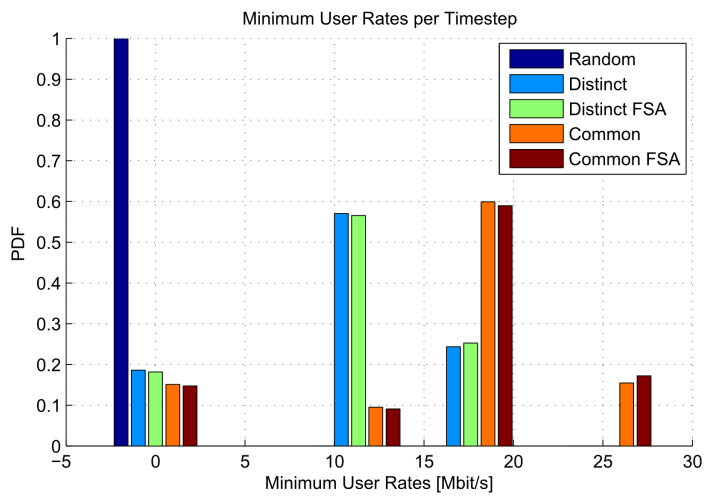

Fig. 9: Minimum user rates without SIC.

\begin{tabular}{|l|l|l|l|l|l|}
\hline \multirow{2}{*}{ FR } & \multirow{2}{*}{ SIC } & \multirow{2}{*}{ Scheduling } & \multicolumn{2}{|c|}{ Capacity [Gbps] } & \multirow{2}{*}{ Availability } \\
\cline { 4 - 5 } & & & Value & Increase & \\
\hline 4 & No & Random & 384.6 & - & $99.99 \%$ \\
\hline \multirow{5}{*}{1} & & Random & 575.7 & $49.7 \%$ & $98.92 \%$ \\
& \multirow{3}{*}{ No } & Distinct & 585.2 & $52.2 \%$ & $99.97 \%$ \\
& & Distinct FSA & 578.4 & $50.4 \%$ & $99.97 \%$ \\
& & Common & 596 & $55 \%$ & $99.97 \%$ \\
\cline { 3 - 5 } & & Common FSA & 586.7 & $52.5 \%$ & $99.97 \%$ \\
\cline { 3 - 5 } & \multirow{2}{*}{ Yes } & Random & 746.2 & $94 \%$ & $99.99 \%$ \\
& & Distinct & 762 & $98.1 \%$ & $99.99 \%$ \\
& & Distinct FSA & 742.9 & $93.2 \%$ & $99.99 \%$ \\
& & Common & 766 & $99.2 \%$ & $99.99 \%$ \\
& & Common FSA & 746.8 & $94.2 \%$ & $99.99 \%$ \\
\hline
\end{tabular}

TABLE II: System capacity and availability improvements.

In this study, the scheduling performance is evaluated in the return link of a full frequency reuse HTS system with and without the application of successive interference cancellation (SIC) at the gateway. A bandwidth of $1.05 \mathrm{GHz}$ in $\mathrm{Ka}$ band is considered in the BATS system with 302 beams over Europe, and the DVB-RCS2 MODCODs are applied. The resulting system capacity and minimum user rates are shown in Fig. 6 and Fig. 7 for the case with SIC, and in Fig. 8 and Fig. 9 for the case without. While the studied scheduling algorithms do not offer a significant increase in the average system capacity as compared to random scheduling in the full FR case, they are designed to increase the minimum user rates. However, it has to be noted that either scheduling or SIC need to be employed so that the system meets the availability requirement of $99.7 \%$. In terms of system capacity, the common merging gives the best results, i.e. the average system capacity is increased by $2.7 \%$ in the case with SIC and by $3.5 \%$ in the case without SIC as compared to the random scheduling method. The distinct method offers a good compromise between performance and computational complexity. In the case with SIC, the FSA algorithm has a performance comparable to the random scheduling, while it is slightly better in the case without SIC. However, it should be noted that the full frequency reuse system with scheduling and SIC is able to double the capacity as compared to the state-of-the-art 4-color scheme with random scheduling, while it still offers more than
$50 \%$ increase, when SIC is not employed. The results for the capacity and availability improvements are summarized in TABLE II. Regarding the minimum user rate, the random scheduling is outperformed by all advanced algorithms. With SIC, all four advanced algorithms show similar performance and increase the minimum user rate from $10 \mathrm{Mbit} / \mathrm{s}$ to more than $30 \mathrm{Mbit} / \mathrm{s}$ for almost all time steps. Without SIC, the common and common FSA algorithms push the minimum user rates above $20 \mathrm{Mbit} / \mathrm{s}$ for more than $75 \%$ of the time, while the distinct and distinct FSA algorithms offer between 10 and 20 $\mathrm{Mbit} / \mathrm{s}$. As a reference, the 4 color reuse system with random scheduling offers only $15 \mathrm{Mbit} / \mathrm{s}$ of minimum user rates for more than $75 \%$ of the time.

\section{CONCLUSION}

In this paper, CCI-aware RRM is shown to be an enabling factor for realization of full resource reuse HTS communication systems. Several algorithms are presented for a centralized setup, and they are tested in a realistic system simulator. Satellite-switched smart gateway diversity with scheduling is studied for the feeder link, and it helps to reduce the total number of gateways in the system, while minimizing the capacity losses and ensuring the necessary feeder link availability in rain fading. In the forward user link, an interference-aware scheduling approach for full frequency reuse increases the achievable user rates and offers $35.7 \%$ increase of the system capacity as compared to state-of-the-art 4-color reuse with random scheduling. Scheduling for full frequency reuse in the return user link based on multi-partite graph matching techniques is shown to even double the system capacity as compared to the 4-color reuse scheme, when interference cancellation is applied at the gateway with minimum user rates above $30 \mathrm{Mbit} / \mathrm{s}$. In the case without SIC, at least $50 \%$ increase of system capacity is expected with minimum user rates of more than $20 \mathrm{Mbit} / \mathrm{s}$ for more than $75 \%$ of the time.

\section{ACKNOWLEDGEMENT}

This work has been supported by the BATS research project funded by the EU-FP7 under contract n 317533 .

\section{REFERENCES}

[1] Broadband Access via Integrated Terrestrial and Satellite Systems (BATS), "ICT-2011.1.1 BATS D4.1: Satellite Network Mission Requirements," European Project, Tech. Rep., 2012.

[2] S. Dimitrov, S. Erl, S. Jaeckel, J. M. Rodriguez, A. Yun, A. Kyrgiazos, B. Evans, O. Vidal, and P. Inigo, "Radio Resource Management for Forward and Return Links in High Throughput Satellite Systems," in in Proc. of 20th Ka and Broadband Communications Conference, Vietri sul Mare / Salerno, Italy, Oct. 1-3 2014.

[3] Second generation framing structure, channel coding and modulation systems for Broadcasting, Interactive Services, News Gathering and other broadband satellite applications (DVB-S2), Digital Video Broadcasting (DVB) Std. ETSI EN 302 307, Aug. 2009.

[4] Second generation framing structure, channel coding and modulation systems for Broadcasting, Interactive Services, News Gathering and other broadband satellite applications; Part —-: S2-Extensions (DVB-S2X) (Optional), Digital Video Broadcasting (DVB) Std. ETSI EN 302307 2, Mar. 2014.

[5] Second Generation DVB Interactive Satellite System (DVB-RCS2); Part 2: Lower Layers for Satellite Standard, Digital Video Broadcasting (DVB) Std. ETSI EN 301 545-2, Jan. 2012. 
[6] A. Kyrgiazos, P. Thompson, B. Evans, A. Kyrgiazos, B. Evans, and P. Thompson, "On the gateway diversity for high throughput broadband satellite systems," Wireless Communications, IEEE Transactions on, vol. 13, no. 10, pp. 5411-5426, Oct 2014.

[7] V. Boussemart, M. Berioli, and F. Rossetto, "User scheduling for large multi-beam satellite MIMO systems," in In Proc. of the IEEE Asilomar Conference on Signals, Systems and Computers (ASILOMAR), 2011, pp. 1800-1804. 